\title{
Multipoint Spectroscopy Measurement of Spherical Tokamak Heating by Magnetic Reconnection in UTST ${ }^{*}$
}

\author{
Shuji KAMIO, Qinghong CAO, Keita ABE, Morio SAKUMURA, Naoto SUZUKI, \\ Takenori G WATANABE, Koji ISHIGUCHI ${ }^{1)}$, Ryota IMAZAWA ${ }^{2)}$, Takuma YAMADA, \\ Michiaki INOMOTO, Yuichi TAKASE and Yasushi ONO \\ Graduate School of Frontier Sciences, The University of Tokyo, Kashiwa 277-8561, Japan \\ ${ }^{1)}$ The Open University of Japan, Mihama 261-8586, Japan \\ 2) Japan Atomic Energy Agency, Naka 311-0193, Japan
}

(Received 6 December 2010 / Accepted 23 February 2011)

\begin{abstract}
The temporal evolution of ion temperature and flow was observed to investigate the heating effect of plasma merging in the UTST device by using a multipoint Doppler spectroscopy measurement system. The bulk plasma ion temperature measured by a carbon impurity line was about $15-20 \mathrm{eV}$ after plasma merging. In addition, anomalous broadening of the carbon line width was observed only in the early phase of plasma merging. Since carbon impurity emission comes mainly from the X-point and the vicinity of the center stack, the broad spectrum is considered to consist of a radially bi-directional outflow component from the X-point and a stationary component near the center stack. The outflow velocity estimated from the spectroscopy was about $24 \mathrm{~km} / \mathrm{s}$, which agreed well with both an Alfvén velocity of $22 \mathrm{~km} / \mathrm{s}$ and a velocity of the reconnected field line motion of $35 \mathrm{~km} / \mathrm{s}$. (c) 2011 The Japan Society of Plasma Science and Nuclear Fusion Research
\end{abstract}

Keywords: Doppler spectroscopy, spherical tokamak, magnetic reconnection, plasma merging

DOI: $10.1585 /$ pfr.6.2402033

\section{Introduction}

The spherical tokamak (ST) is an attractive candidate for a fusion core plasma because of its good confinement, good stability, and high-beta properties. The beta value is the ratio of plasma thermal pressure to magnetic pressure and is often used to evaluate the efficiency of a magnetic confined fusion reactor. The ST's low aspect ratio and non-circular cross section are suitable for confining high-beta plasmas; however, high-power external heating systems such as neutral beam injection or radio frequency waves are required to form a high-beta equilibrium.

The plasma merging method is a novel startup scheme for forming a high-beta equilibrium in a short period. Plasma merging induces magnetic reconnection, which is rapid conversion from magnetic energy to plasma kinetic or thermal energy, between two initial torus plasmas. Thus, plasma merging increases the plasma thermal energy and decreases the magnetic energy, dramatically increasing the beta value. The merging startup method has been demonstrated in the START/MAST [1] (UKAEA) and TS-3/4 (The University of Tokyo) devices. In TS-3, two STs were merged to form a single ST with a beta of up to 50\% [2], and an oblate field-reversed plasma formed by counterhelicity merging was transformed into an ultra-high-beta (up to $80 \%$ ) ST by applying an external toroidal field after merging $[3,4]$. The high-beta value obtained in these

author'se-mail:kamio@ts.t.u-tokyo.ac.jp

*) This article is based on the presentation at the 20th International Toki Conference (ITC20). experiments come mainly from significant ion heating by magnetic reconnection. Doppler spectroscopy measurements in TS-3 showed that ions are heated from $10 \mathrm{eV}$ to $40-60 \mathrm{eV}$ in $10 \mu \mathrm{s}$, which corresponds to a heating power of as large as 4-6 MW [5]. However, in these previous experiments, plasma merging startup was performed by using poloidal field (PF) coils inside the vacuum vessel, which cannot be used in future fusion reactors. The University of Tokyo Spherical Tokamak (UTST) [6] was constructed to investigate the feasibility of merging startup of high-beta STs under a more reactor-relevant condition with no internal PF coils. To confirm the formation of a high-beta ST plasma in the UTST, ion-temperature measurement is urgently required. This paper describes the development of an ion-temperature/flow measurement system using multipoint Doppler spectroscopy and its initial results for merging startup in the UTST.

\section{The UTST Plasma Merging Device}

A high-beta ST plasma is formed by double null merging (DNM) in the UTST. In the DNM startup method, two initial STs are generated at null points located on both ends of the device, and they are merged to form an ST, as illustrated in Fig. 1. During plasma merging, the tension force of the reconnected magnetic field lines accelerates the plasma to the Alfvén velocity. The accelerated ions are thermalized in the downstream area [5] by damping mechanisms such as viscosity or fast shock structure, resulting 


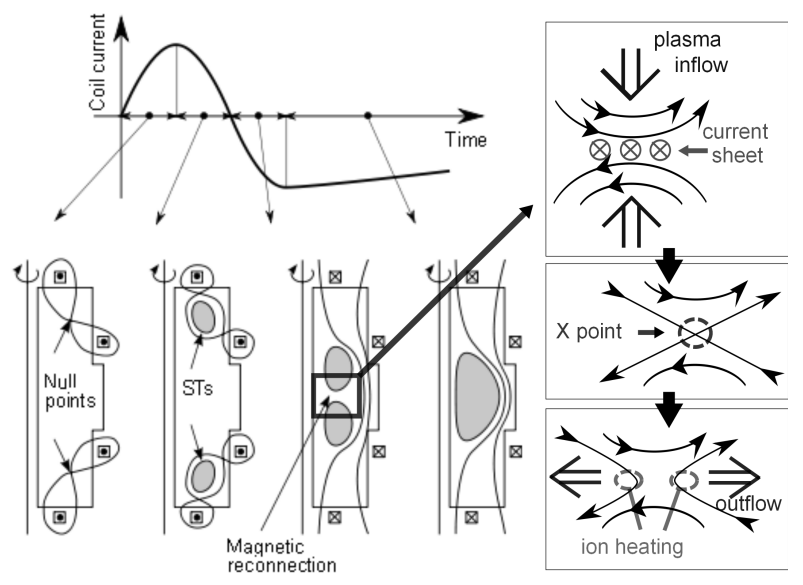

Fig. 1 Schematic view of high-beta ST startup by DNM in the UTST.

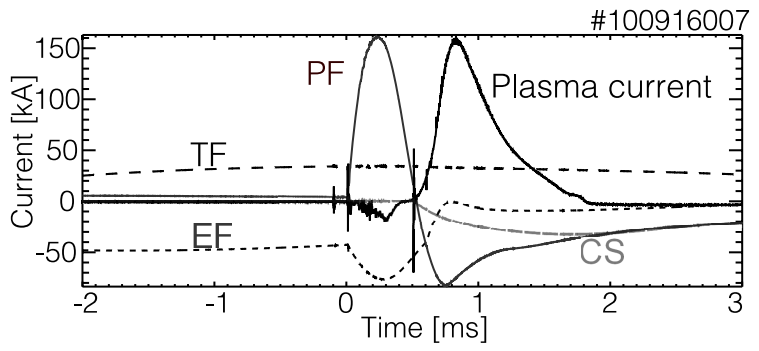

Fig. 2 Typical waveforms of coil currents and plasma during DNM startup in the UTST.

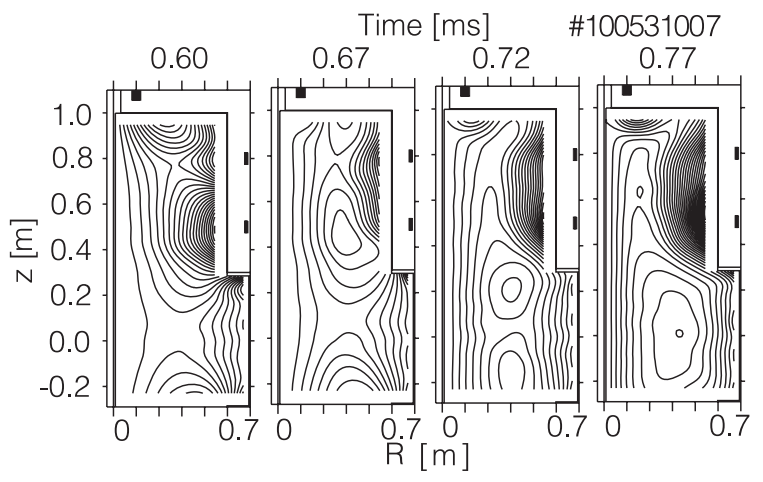

Fig. 3 Evolution of poloidal flux surfaces during DNM startup.

in the formation of a high-beta ST equilibrium. In the UTST device, all the coils are located outside the vacuum vessel, whereas all the other existing merging devices use internal coils to form the initial plasmas. Fig. 2 shows the typical discharge waveforms of DNM startup. The swing of the PF coils applies a parallel electric field at the null points to initiate the torus discharge and drive the plasma current. The toroidal field (TF) coil, equilibrium field (EF) coils, and PF coils start to discharge at $-4.3,-6$, and $0 \mathrm{~ms}$, respectively. The plasma current is as high as $160 \mathrm{kA}$, and the pulse width is as great as $1.2 \mathrm{~ms}$ with the assistance of the center solenoid (CS) coil, which begins discharging at $0.5 \mathrm{~ms}$.
Fig. 3 shows the poloidal flux surfaces during plasma merging measured by a two-dimensional (axial: $z$ and radial: $R$ ) magnetic pickup coil array. The array has 128 channels in the upper section around the null point and 162 channels in the central section to measure the magnetic field $B_{\mathrm{t}}$ and $B_{z}$ directly inside the vacuum vessel. The poloidal flux $\Psi$ and toroidal current density $j_{\mathrm{t}}$ can be derived from the measured magnetic field.

\section{Development of the Spectroscopy Measurement System}

The Doppler shift $\Delta \lambda$ of light emitted from a traveling particle is defined in terms of the particle's velocity as $\Delta \lambda=\left(\lambda_{0} v\right) / c$, where $v$ is the particle's velocity, $c$ is the speed of light, and $\lambda_{0}$ is the wavelength of the emitted line. Doppler spectroscopy measurement applies this principle to diagnose the behavior of ions in plasma. The velocity distribution determined by the thermal and collective motions yields the emission line intensity $I$ in Gaussian form as

$$
\begin{aligned}
& I(\Delta \lambda)=\frac{I_{0}}{\pi^{1 / 2} \Delta \lambda_{\mathrm{D}}} \exp \left\{-\left(\frac{\Delta \lambda}{\Delta \lambda_{\mathrm{D}}}\right)^{2}\right\}, \\
& \Delta \lambda_{\mathrm{D}}=\frac{\lambda_{0}}{c} \sqrt{\frac{2 k T_{\mathrm{i}}}{M_{\mathrm{i}}}},
\end{aligned}
$$

where $T_{\mathrm{i}}$ is the ion temperature and $M_{\mathrm{i}}$ is the ion mass. The temperature is calculated by the full width at half maximum (FWHM) of the line broadening:

$$
\begin{aligned}
\Delta \lambda_{\mathrm{FWHM}} & =2(\ln 2)^{1 / 2} \Delta \lambda_{\mathrm{D}} \\
& =7.69 \times 10^{-5} \lambda_{0}\left(\frac{T_{\mathrm{i}}}{A}\right)^{1 / 2}, \\
T_{\mathrm{i}}=1.7 & \times A \times 10^{8}\left(\frac{\Delta \lambda_{\mathrm{FWHM}}}{\lambda_{0}}\right)^{2} .
\end{aligned}
$$

The ion flow velocity is calculated by the Doppler shift of the line peak.

Fig. 4 shows the arrangement of the lines of sight. To measure the ion acceleration and local heating caused by magnetic reconnection, we added a radial path that directly sees the center stack in addition to the commonly used tangential paths (chords). Ion emission along the lines of sight is introduced to two monochromators $(1 \mathrm{~m}$, 1200 grooves $/ \mathrm{mm}$ ). Each diffracted light beam is magnified and focused on the detector by five lenses, and then detected by a 16-channel photomultiplier tube (PMT) array (Hamamatsu R5900U-01-L16). The PMTs have a highspeed response with a $0.6 \mathrm{~ns}$ rise time, so we can measure the time evolution of the plasma ion temperature and flow during magnetic reconnection. To determine the spatial distribution of light emission or Doppler broadening, we combined the measured results from 13 discharges, assuming good reproducibility. We derived the local iontemperature profile from the measured spectra of lineintegrated emission by using an Abel inversion technique. 


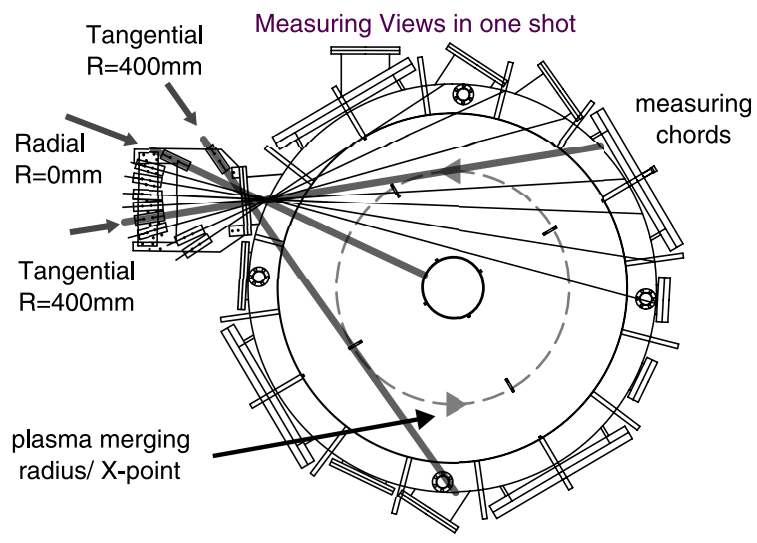

Fig. 4 Alignment of lines of sight in Doppler spectroscopy measurement, which has a radial view and nine tangential views on a $z=0$ cross section of the UTST.
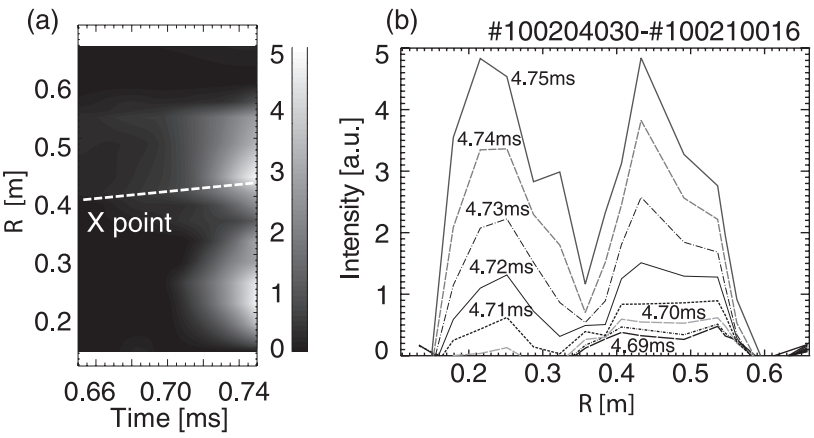

Fig. 5 (a) CII $(426.7 \mathrm{~nm})$ line intensity profile after Abel transformation during plasma merging, (b) evolution of radial intensity profiles.

A carbon impurity line (CII, $426.7 \mathrm{~nm}$ ) was used in this research.

\section{Results and Discussion}

Figure 5 (a) shows the time evolution of the CII impurity line intensity. Assuming toroidal symmetry, the local intensity $f(r)$ is calculated from the integrated intensity $F$ by

$$
f(r)=-\frac{1}{\pi} \int_{r}^{R} \frac{\mathrm{d} F}{\mathrm{~d} y} \frac{\mathrm{d} y}{\sqrt{y^{2}-r^{2}}} .
$$

After plasma merging, strong emission from the $R<0.2 \mathrm{~m}$ region appears, possibly due to carbon impurities supplied from the center stack. Plasma merging occurs around $0.7 \mathrm{~ms}$, just before the bright emission from the center stack appears, as seen in Fig. 3. During plasma merging, emission from carbon impurities is observed at two distinct locations: near the X-point and near the center stack. Fig. 5 (b) shows the time evolution of the intensity profiles. The carbon impurity emission initially comes from the Xpoint region at $0.69 \mathrm{~ms}$, and then the emission from the center stack grows. Thus, the spectrum from the radial

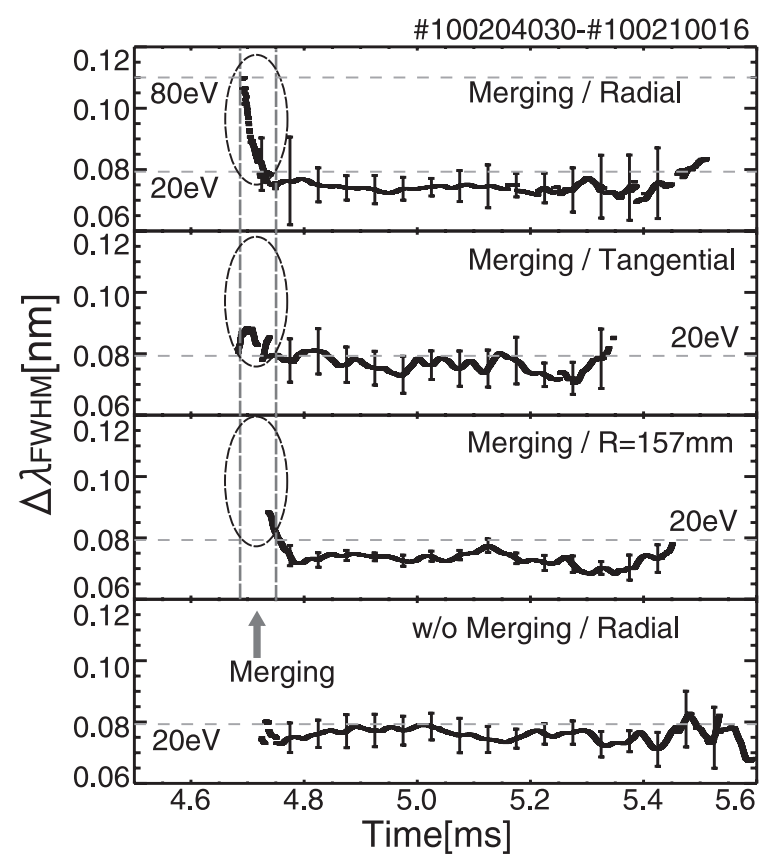

Fig. 6 Time evolution of carbon line broadening observed in various views and types of plasma operation.

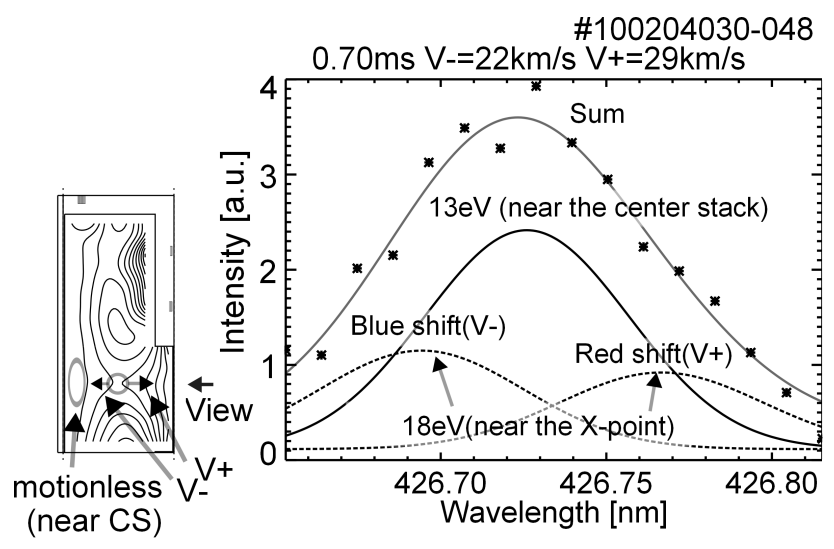

(a)

(b)

Fig. 7 (a) Contour of poloidal flux surface at plasma merging, (b) broadened spectrum of carbon impurity line and its fitting using three Gaussian profiles.

viewing path reflects the ion behavior near the $\mathrm{X}$-point and center stack.

Fig. 6 shows the time evolution of the spectrum broadening width in the cases with and without plasma merging. The error bar shows the shot-by-shot dispersion of the results from five discharges. The ion temperature is $15-20 \mathrm{eV}$ after $0.9 \mathrm{~ms}$ regardless of the type of operation, and a significant temperature increase was not confirmed. However, anomalous spectral broadening $\Delta \lambda_{\text {FWHM }}$ was observed only in the radial view early in plasma merging. Since this wide broadening consists of emission components from the X-point and the center stack, the broadening does not necessarily correspond to the ion thermal temperature. The emission from the $\mathrm{X}$-point is expected to 
include the effect of magnetic reconnection, which generates the bi-directional radial outflow. Hence, we assumed that the wide broadening is a mixture of three Gaussians with different shifts, as shown in Fig. 7. The three different shifts are assumed to come from two accelerated components in the magnetic reconnection outflows and a stationary component of the plasma near the center stack. The broadened spectrum is fitted as shown in Fig. 7 (b) so as to minimize the error between the sum of the three Gaussian profiles and the measurement by adjusting the center wavelength, broadening and intensity of the Gaussian on the basis of the ratio of intensity profiles shown in Fig. 5 (b). The fitted Doppler broadening of the ion temperature was $18 \mathrm{eV}$ (near the X-point) and $13 \mathrm{eV}$ (near the center stack). This fitting allows us to estimate the radial velocity of carbon ions accelerated by magnetic reconnection. The estimated outflow velocity is about $24 \mathrm{~km} / \mathrm{s}$ early in the reconnection and then decreases.

The outflow velocity owing to magnetic reconnection is considered to reach the Alfvén velocity $v_{\mathrm{A}}$ [7] using the upstream poloidal magnetic field and downstream plasma density,

$$
v_{\mathrm{A}}^{2}=\frac{B_{0}^{2}}{\mu_{0} \rho_{\mathrm{m}}},
$$

where $B_{0}$ is the inflow poloidal magnetic field, and $\rho_{\mathrm{m}}$ is the mass density in the outflow region. The Alfvén velocity $v_{\mathrm{A}}$ of the plasma merging experiment in the UTST is about $22 \mathrm{~km} / \mathrm{s}$, which is close to the outflow velocity estimated from Doppler spectroscopy.

The outflow velocity is also estimated from the speed of magnetic field line motion measured by the twodimensional magnetic probe array. The field line velocity is about $35 \mathrm{~km} / \mathrm{s}$, which is again close to the outflow velocity calculated from the Doppler shift and the Alfvén velocity.

The energy relaxation time constants of energetic carbon ions by electrons $\left(\tau_{\mathrm{E}}^{\mathrm{Ce}}\right)$ and hydrogen ions $\left(\tau_{\mathrm{E}}^{\mathrm{CH}}\right)$ are given as follows [8]:

$$
\begin{gathered}
\tau_{\mathrm{E}}^{\mathrm{Ce}}=\frac{6 \pi \sqrt{2 \pi} \varepsilon_{0}^{2} m_{\mathrm{C}} m_{\mathrm{e}}^{-1 / 2} T_{\mathrm{e}}^{3 / 2}}{Z_{\mathrm{C}}^{2} n_{\mathrm{e}} e^{4} \ln \Lambda} \approx 3.7 \mathrm{~ms}, \\
\tau_{\mathrm{E}}^{\mathrm{CH}}=\frac{m_{\mathrm{H}}}{m_{\mathrm{C}}+m_{\mathrm{H}}} \frac{2 \pi \varepsilon_{0}^{2} m_{\mathrm{C}}^{2} 2 v_{\text {outflow }}^{3}}{n_{\mathrm{H}} e^{4} \ln \Lambda} \approx 21 \mu \mathrm{s} .
\end{gathered}
$$

The carbon-hydrogen energy relaxation time $\tau_{\mathrm{E}}^{\mathrm{CH}}$ is close to the observed decay time of the outflow kinetic energy, $17 \mu$ s, in Fig. 8. Thus, the accelerated carbon ions are slowed quickly by energy relaxation with hydrogen ions in the plasma. This indicates that the released energy is not large enough to heat the entire plasma under the present experimental conditions.

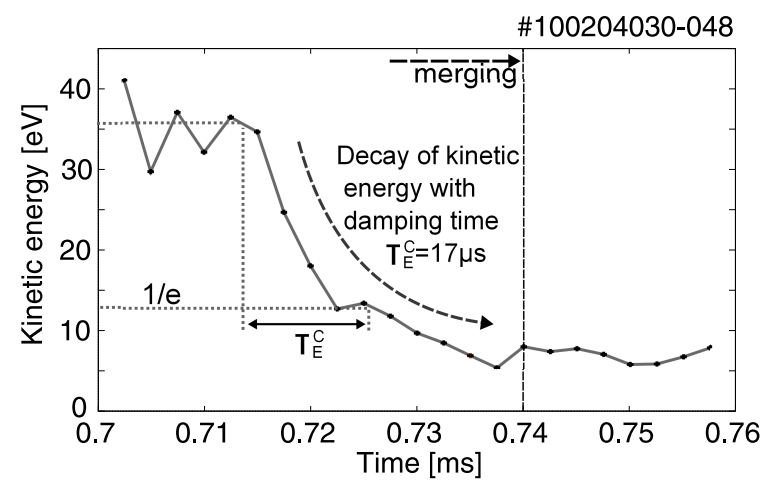

Fig. 8 Time evolution of the kinetic energy of accelerated carbon ions.

\section{Conclusion}

A multipoint Doppler spectroscopy system using a multichannel PMT array and matrix was developed and installed at the UTST. The bulk plasma ion temperature measured by a carbon impurity line (CII, $426.7 \mathrm{~nm}$ ) was about $15-20 \mathrm{eV}$. Although a temperature increase was not confirmed, radial acceleration of carbon ions was observed during plasma merging. The flow velocity was estimated to be about $24 \mathrm{~km} / \mathrm{s}$, which is close to the Alfvén velocity of $22 \mathrm{~km} / \mathrm{s}$ and the velocity of magnetic field lines of $35 \mathrm{~km} / \mathrm{s}$, indicating that carbon ions are accelerated by magnetic reconnection in the UTST. We successfully demonstrated energy conversion through magnetic reconnection by plasma merging in the UTST. The increment of the plasma current of the initial STs increases the magnetic energy release, making more effective plasma heating possible.

\section{Acknowledgments}

We are deeply grateful to Prof. Shigefumi Okada, who assisted us with the equipment and instruments. Special thanks also go to Dr. Yoichi Hirano, Dr. Satoru Kiyama, Dr. Hajime Sakakita, and Dr. Haruhisa Koguchi, who provided carefully considered feedback and valuable comments. This work was supported by Grants-in-Aid for Scientific Research, JSPS, Japan (22246119, 22686085, and 21760688) and the Global COE Program "Secure-Life Electronics," MEXT, Japan.

[1] A. Sykes et al., Nucl. Fusion 41, 1423 (2001).

[2] Y. Ono et al., Phys. Plasmas 4, 1953 (1997).

[3] Y. Ono and M. Inomoto, Phys. Plasmas 7, 1863 (2000).

[4] Y. Ono et al., Nucl. Fusion 43, 789 (2003).

[5] Y. Ono et al., Fusion energy 2008 (IAEA Vienna, 2009, in DVD-ROM) EX/P9-4.

[6] T. Yamada et al., Plasma Fusion Res. 5, S2100 (2010).

[7] E. N. Parker, J. Geophys. Res. 62, 509 (1957).

[8] J. Wesson, Tokamaks 3rd Edition (Oxford University Press on Demand, 2004) pp.246-253. 